\title{
EL NARRADOR DRAMATIZADO DE JOSEPH ANDREWS Y LE ROMAN COMIQUE
}

Isabel Medrano Vicario

UNED

El poderoso influjo que el narrador de las novelas de Fielding ejerce sobre el bien intencionado lector ha constituido tradicionalmente para la crítica uno de los puntos clave de su peculiar estilo discursivo. Convencido, como muchos de sus contemporáneos, de la doble función de la literatura, que debe perseguir por igual la enseñanza y el deleite, el autor de Tom Jones y Joseph Andrews procurará por todos los medios transmitir al lector sus convicciones morales y sus ansias de renovación social; su didacticismo, sin embargo, queda considerablemente suavizado en un relato dotado de unas grandes dosis de humor e ironía.

La obstinada omnipresencia de un narrador que hace gala de un conocimiento exhaustivo no sólo de los hechos relatados, sino hasta de los impulsos más íntimos de los personajes, y que se permite interrumpir continuamente el relato para exponer sus propias opiniones ha convertido a Fielding en uno de los exponentes máximos de este tipo de narración autoritaria. Este narrador hace uso de una calculada estrategia con el fin de estimular la participación del lector y provocar en él una respuesta determinada que deberá poner en cuestión las normas establecidas; tal figura representa, pues, el vehículo fundamental del afán didáctico de un autor que ha establecido un estudiado plan en el que todas las partes integrantes, incluido el receptor de su mensaje, juegan un papel señalado de antemano.

Este papel privilegiado del narrador constituye una práctica literaria tan antigua como la épica griega. Este género, típico, como afirma Lukacs, de una forma de sociedad cerrada en sí misma, que defiende unos valores colectivamente reconocidos de los cuales son portadores 
los héroes, utilizaba un recurso semejante. El marcado carácter didáctico de esta forma literaria -el conocimiento de la epopeya constituía uno de los métodos principales de educación de la juventud en tiempos de Platón- residía, sobre todo, en el hecho de que el narrador basaba su historia en temas conocidos por el auditorio, lo que concede a este género un carácter objetivo de que carecen otras formas poéticas o narrativas. Existían una serie de tópicos extraídos del acervo popular que el poeta épico, inspirado por la Memoria, madre de las Musas, debía recordar; este conocimiento, de algún modo superior, que hundía sus raíces en la leyenda y en el mito, le confería una posición privilegiada: era el cantor de los dioses, y su voz estaba sancionada por la divinidad; hablaba como vate, como iniciado y su tono se caracterizaba por una cierta solemnidad y dignidad.

En opinión de Delasanta' el lector atento puede notar la constante presencia del autor en las obras homéricas por sus continuas referencias a las Musas, sus explicaciones subjetivas de ciertos acontecimientos, por las reacciones del poeta, favorables o desfavorables, ante los hechos que narra, a veces incluso porque el narrador se dirige directamente al personaje que ocupa el centro del relato.

Aparte de estas continuas intervenciones personales, Delasanta argumenta que la prueba más evidente de la omnisciencia homérica es su visión panorámica del mundo, esa posición privilegiada que le hace conocedor de la totalidad de los hechos, independientemente del tiempo y el lugar en que ocurran. Es esta visión panorámica, sin duda, la que le permite todo tipo de intromisiones en la acción dramática y el uso de recursos estilísticos como la anticipación, el proceso retardador, las interpolaciones, o la composición simétrica o circular.

También Booth ${ }^{2}$ señala cómo en la Ilíada y en la Odisea pueden apreciarse las continuas intromisiones del autor, que explica los motivos de algunos personajes o la relevancia de ciertos acontecimientos, en un afán de controlar las creencias, las simpatías o los intereses del receptor de su mensaje; así, mediante breves comentarios intercalados en la historia, se nos informa sobre la inocencia o culpabilidad, la indiscreción o el buen juicio, el heroísmo o la cobardía de los personajes con unos niveles de fiabilidad que sólo un narrador semejante puede ofrecernos.

Scholes y Kellog ${ }^{3}$, al tratar el punto de vista en la narración, aportan un nuevo elemento clarificador en su análisis de la postura omnis-

I R. Delasanta, The Epic Voice, The Hague-Paris, Mouton, 1967, pp.39-40.

2 W.C. Booth, La retórica de la ficción, Barcelona, Bosch, 1974, pp. 4-6.

3 R. Scholes \& R. Kellog, The Nature of Narrative, New York, Oxford University Press. 1966, pp. 266-267. 
ciente del narrador de Joseph Andrews y Tom Jones. Su autoridad no es únicamente reflejo de la que poseía el bardo épico, sino también de la del historiador clásico griego, cuya función no se reducía a relatar los acontecimientos pasados, sino a intentar descubrir la verdad de los mismos, separando la historia del mito. El espíritu crítico de que hacen gala historiadores como Heródoto o Tucídides les confiere una autoridad que les permite no sólo presentar los hechos acaecidos en la forma que consideran más adecuada, sino incluir comentarios al respecto, moralizar, generalizar, encauzar la opinión del lector e incluso aconsejarle.

Como afirman estos críticos, la historia en sus comienzos se hallaba íntimamente unida a la retórica y el historiador sabía que una de sus principales tareas consistía en convencer al auditorio de que poseía la autoridad y competencia necesarias para tratar aquellos temas que eran objeto de su estudio. Cuando la Europa de la Ilustración confirió al tratamiento de la historia un mayor rigor, los historiadores tuvieron que desprenderse de este privilegio de la omnisciencia, del que rápidamente se adueñó la naciente narración literaria. Según Scholes y Kellog, la combinación por parte de Fielding de estas dos fuentes de autoridad narrativa posee una importancia decisiva para la historia de la novela, no tanto por haber integrado en su obra lo ficticio y lo empírico, como había hecho Cervantes con anterioridad, sino porque las justificaciones teóricas producto de esta práctica constituyen un importante documento crítico.

El gran conocedor de los clásicos que fue Fielding pudo sin duda tomar de los escritores de la antigüedad este recurso, que él desarrolló y perfeccionó hasta convertirlo en uno de los más característicos de su estilo literario. Existen, sin embargo, otras fuentes más cercanas en el tiempo que contribuyeron a consolidar su arte narrativo; la lectura de autores como Cervantes, Scarron, Lesage y Marivaux -citados de forma elogiosa en Joseph Andrews por considerar que todos ellos habían contribuido a establecer las bases de la auténtica creación literaria-, resultó evidentemente decisiva en la elaboración de una teoría apenas esbozada en esta novela pero ya consolidada de forma magistral en Tom Jones.

Es naturalmente en su primera novela, Joseph Andrews, donde se aprecian más claramente ciertos paralelismos en el uso de algunos recursos tomados de los autores citados. La figura del narrador autoritario había sido utilizada por Scarron en Le Roman Comique -y anteriormente por Cervantes- en una estrategia similar a la empleada por Fielding. Este estudio trata de mostrar dicho paralelismo, producto sin duda de una concepción semejante de la obra literaria. 
Seymour Chatman 4 establece una serie de constantes indicativas de la presencia del narrador omnisciente, que él denomina "overt narrator"; la mayoría de estas constantes, casi todas ellas senaladas anteriormente por Booth en su Rhetoric of Fiction, aunque de manera menos sistematizada, aparecen en las novelas que nos ocupan. Los comentarios implícitos o explícitos acerca de los hechos narrados constituyen para Chatman, por su gratuidad, el elemento más significativo del autoritarismo del narrador; los comentarios implícitos tienen carácter irónico y los explícitos pueden hacer referencia a la trama -interpretando los hechos, emitiendo juicios morales o incluyendo generalizaciones que relacionen la ficción con el mundo real- o referirse al discurso.

Los comentarios implícitos son más abundantes en Joseph Andrews, obra en que la ironía juega un papel más importante que en Le Roman Comique, donde predomina el humor. La ironía, por otra parte, es un recurso complejo con múltiples manifestaciones; haré referencia únicamente a la forma más elemental, en que la comunicación entre narrador y lector se lleva a cabo mediante manifestaciones que poseen un sentido diferente del que aparentan, siempre a costa de un tercer elemento respecto al cual se ironiza.

Los elementos contra los que ironizan Fielding y Scarron son semejantes:

- las costumbres sociales de sus épocas respectivas, denunciadas bien directamente o sirviéndose de un personaje de segundo orden cuya conducta resulta criticable. Son sobre todo las clases altas las que merecen esta crítica:

\begin{abstract}
"they could not however teach him to game, swear, drink, nor any other genteel Vice the Town abounded with" (Joseph Andrews, 1.4) "for as we are now drawing near the Seat of the Boobies; and as that is a ticklish Name, which malicious persons may apply according to their evil Inclinations to several worthy Country 'Squires, a Race of Men whom we look upon as entirely inoffensive, and for whom we have an adequate Regard, we shall lend no assitance to any such malicious Purposes" (J.A., III.2)

"Enfin, il se tint à la fille d'un marquis de je ne sais quel marquisat; car c'est la chose du monde dont je voudrais le moins jurer en un temps où tout le monde se marquise de soi-mème, je veux dire de son chef" (Le Roman Comique, I.9)
\end{abstract}

4 S. Chatman, Story and Discourse, Ithaca \& London, Cornell University Press, 1978, pp. 228 y sig. 
- ciertos profesionales poco escrupulosos que ejercen de forma indebida su función. Este tipo de crítica impregna varios pasajes de Joseph Andrews y va dirigida especialmente a médicos, abogados y eclesiásticos:

“...the Lawyer, who was afraid of some Mischief happening to himself if the Wretch was left behind in that Condition..." (J.A., I.12)

También se detecta en Scarron, aunque tiene un carácter más suave:

"Ce curé donc, ...fit consulter sa gravelle par les médecins du Mans qui lui dirent, en latin fort élégant, qu'il avait la gravelle (ce que le pauvre homme ne savait que trop)..." (R.C., I.14)

"On a voulu dire qu'il jura Dieu, mais je ne puis croire cela d'un curé du bas Maine" (R.C., I.14)

- algunos hombres de letras contemporáneos y sus creaciones, o ciertos usos literarios en boga:

“...Simplicity was his [Adams'] Characteristic: he did, no more than Mr. Colley Cibber, apprehend any such Passions as Malice and Envy to exist in Mankind, which was indeed less remarkable in a Country Parson than in a Gentleman who hath past his Life behind the Scenes, a Place which hath been seldom thought the School of Innocence; and where a very little Observation would have convinced the great Apologist, that those Passions have a real Existence in the human Mind" (J.A., 1.3).

"Before we proceed any farther in this Tragedy, we shall leave Mr. Joseph and Mr. Adams to themselves, and imitate the wise Conductors of the Stage; who in the midst of a grave Action entertain you with some excellent piece of Satire or Humour called a Dance. Which piece indeed is therefore danced, and not spoke, as it is delivered to the Audience by Persons whose thinking Faculty is by most People held to lie in their Heels; and to whom, as well as Heroes, who think with their Hands, Nature hath only given Heads for the sake of Conformity, and as they are of use in Dancing, to hang their Hats on" (J.A., III.10) "Je ne vous dirai point exactement s'il avait soupé et s"il se coucha sans manger, comme font quelques faiseurs de romans que règlent toutes les heures du jour de leurs héros, les font lever de bon matin, conter 
leur histoire jusqu'à l'heure du dîner, dîner fort légèrement et après diner reprendre leur histoire ou s'enfoncer dans un bois pour y parler tout seul, si ce n'est quand ils ont quelque chose à dire aux arbres et aux rochers; à l'heure du souper, se trouver à point nommé dans le lieu où l'on mange, où ils soupirent et rêvent au lieu de manger, et puis s'en vont faire des châteaux en Espagne sur quelque terrasse qui regarde la mer, tandjs qu'un écuyer révèle que son maître est un tel, fils d'un roi tel et qu'il n'y a pas un meilleur prince au monde et qu'encore qu'il soit pour lors le plus beau des mortels, qu'il était encore tout autre chose devant que l'amour l'eût défiguré" $(R . C ., 1.9)$

"Je ne vous dirai point si les flambeaux que tenaient les demoiselles étaient d'argent; c'est pour le moins: ils étaient plutot de vermeil doré ciselé, et la salle était la plus magnifique du monde et, si vous voulez, aussi bien meublée que quelques appartements de nos romans, comme le vaisseau de Zelmandre dans le Polexandre, le palais d'Ibrahim dans l'Illustre Bassa, ou la chambre où le roi d'Assyrie recut Mandane, dans le Cyrus, qui est sans doute, aussi bien que les autres qu j'ai nommés, le livre du monde le mieux meublé" (R.C., I.9)

- el mayor número de comentarios irónicos va dirigido a ciertos personajes, generalmente secundarios, cuya conducta resulta moralmente inaceptable:

"We hope therefore, a judicious Reader will give himself some Pains to observe, what we have so greatly laboured to describe, the different Operations of this Passion of Love in the gentle and cultivated Mind of the Lady Booby, from those which it effected in the less polished and coarser Disposition of Mrs. Slipslop" (J.A., I.7)

"He [Beau Didapper] was not entirely ignorant: For he could talk a little French, and sing two or three Italian Songs: He had lived too much in the World to be bashful, and too much at Court to be proud: He seemed not too much inclined to Avarice; for he was profuse in his Expences: Nor had he all the Features of Prodigality; for he never gave a Shilling: No Hater of Women; for he always dangled after them; yet so little subject to Lust, that he had, among those who Knew him best, the Character of great Moderation in his Pleasures. No Drinker of Wine; nor so addicted to Passion, but that a hot Word or two from an Adversary made him immediately cool" (J.A., IV.9)

“... il [Ragotin] ne put aussi empêcher qu'elle ne lui donnât un si grand coup sur la tête que tout le vaste siège de son étroite raison en fut ébranlé" (R.C., II.7)

"Le Destin rougissait aussi, mais de pudeur, au lieu que la Bouvillon, qui n'en avait plus, rougissait je vous laisse à penser de quoi" (R.C., II.10) 
Entre los comentarios explícitos son las interpretaciones de los hechos relatados los que poseen un carácter más heterogéneo. Las explicaciones del narrador incluyen gran variedad de matices y se refieren tanto a los acontecimientos de la historia como a los personajes, sus motivos y reacciones, hábitos sociales, etc.

He aquí una relación de los diversos tipos de comentario interpretativo frecuentes en las dos obras objeto de este estudio y algunos ejemplos de los mismos:

- son numerosas las conjeturas del narrador acerca de los acontecimientos o actitudes de los personajes:

"indeed it is probable, Mr. Adms had rescued more than Fanny from the Danger of a Rape that Evening" (J.A., II.13)

"and indeed before he could well defend himself or return the first Blow, he received a second, which had it fallen on that part of the Stomach to which it was directed, would have been probably the last he would have had any Occasion for" (J.A., IV.7)

"S'il eût été de l'humeur de dom Quichotte, il eût trouvé là de quoi s'en donner jusqu'aux gardes et il se fût cru pour le moins Esplandian ou Amadis" (R.C., I.9)

"Le silence régnait dans l'hôtellerie; les chiens y dormaient, puisqu'ils n'aboyaient point; tous les autres animaux y dormaient aussi ou le devaient faire" (R.C., II.7)

- a menudo se explican o justifican los motivos de la actuación de un personaje o su reacción ante un hecho:

“...a little before the Journey to London, she had been discarded by Mrs. Slipslop on account of her extraordinary Beauty: for I never could find any other reason" (J.A., I.11)

"Joseph saw the Uneasiness she suffered, and immediately removed his Eyes from an Objetc, in surveying which he had felt the greatest Delight which the Organs of Sight were capable of conveying to his Soul. So great was his Fear of offending her, and so truly did his Passion for her deserve the Name of Love" (J.A., IV.7)

"et, pour moi, je crois que, s'il lui eût nommé Angélique ou sa mère La Caverne, qu'il eût oublié le coup de busc de l'une et l'âge de l'autre et se serai donné corps et âme à celle que La Rancune lui aurait nommée, tant le bouquin avait la conscience troublé" $(R . C$., I.11) 
'Le Destin et Léandre ne s'émurent pas beaucoup du conte que le curé leur donnait pour bon, soit qu'ils ne le trouvassent pas si plaisant qu'il leur avait dit, ou qu'ils ne fussent pas alors en humeur de rire" (R.C., II.6)

- el narrador se sirve a veces de una imagen plástica para aclarar ciertas situaciones:

"He was indeed one of the largest Men you should see, and could have acted the part of Sir John Falstaff without stuffing" (J.A., II.14)

"Un petit ours nouveau-né, qui n'a point encore été léché de sa mère, est plus formé en sa figure oursine que ne le fut Ragotin en sa figure humaine après que les piqûres des mouches l'eurent enflé depuis les pieds jusqu'à la tête" (R.C., II.16)

- son abundantes las interpretaciones humoristicas incluidas a lo largo del relato:

\begin{abstract}
"Those who have read any Romance or Poetry antient or modern, must have been informed, that Love hath Wings; by which they are not to understand, as some young Ladies by mistake have done, that a Lover can fly" (J.A., I.11)

"ils renverserent une table à tréteaux qui soutenait la machine harmonieuse et je ne voudrais pas jurer que quelques-uns de ces maudits chiens ne levassent la jambe et ne pissassent contre les orgues renversées, ces animaux étant fort diurétiques de leur nature, principalement quand quelque chienne de leur connaissance a envie de procéder à la multiplication de son espece" (R.C., I.15)

"Le visage de cette nymphe tavernière était le plus petit et son ventre était le plus grand du Maine, quoique cette province abond en personnes ventrues. Je laisse aux naturalistes le soin d'en chercher la raison aussi bien que de la graisse des chapons du pays" (R.C., II.6)
\end{abstract}

- el grado máximo de omnisciencia por parte del narrador lo constituyen las explicaciones relativas al pensamiento o intenciones de los personajes o a hechos que nunca se llevaron a cabo: 
"the old Gentleman abovementioned, thinking the naked Man would afford him frequent Opportunities of shewing his Wit to the Lady..." (J.A., I.12)

"Adams, who knew no more of all this than the Cat which sat on the Table, imagining Mrs. Slipslop's Memory had been much worse than it really was..." (J.A., II.13)

"Ce malheur-là fut cause d'un grand bien, car vraisemblablement il était au plus haut point de sa colère, qui eût sans doute produit un effet digne d'elle si son chapeau, qui le suffoquait, ne l'eût fait songer à sa conservation plutôt qu'à la destruction d'un autre" (R.C., I.10)

"Il eút exagéré la méchante action plus qu'il ne fit s'il eât eu moins de sang à cracher qu'il n'en avait .... (R.C., II.7)

Los juicios de valor, otra de las formas del comentario explícito, ofrecen una evaluación moral de los diferentes personajes y tienen por objeto encauzar las convicciones éticas del lector. Las opiniones del narrador son por lo general favorables cuando hacen referencia a personas de conducta aceptable, y negativas cuando se refieren a personas que el lector debe rechazar; los personajes resultan así extremadamente buenos o malos, sobre todo en la novela de Scarron (Fielding traza caracteres más realistas, como el de Parson Adams, que, aunque eminentemente bueno, comete fallos propios de cualquier ser humano).

Cito a continuación algunos comentarios valorativos expresados por los narradores de Joseph Andrews y Le Roman Comique:

- referidos al carácter de los personajes:

"He [Adams] was ... a Man of good Sense, good Parts, and good Nature; but was at the same time as entirely ignorant of the Ways of this World, as an Infant just entered into it could possibly be" (J.A., I.3) “...my Lady was a Woman of Gaiety, who had been bless'd with a Town Education, and never spoke of her Country Neighbours, by any other Appellation than that of The Brutes" (J.A., I.3)

"Ce n'est pas qu'elle fút dévergondée; mais son humeur enjouée et libre l'empêchait d'observer beaucoup de cérémonies; d'ailleurs, elle avait de l'esprit et était très honnête fille" $(R . C .$, I.8)

“...il était menteur comme un valet, présomptueux et opinâtre comme un pédant et assez mauvais poz̀te pour être étouffé s'il y avait de la police dans le royaume" $(\boldsymbol{R} . \boldsymbol{C}$., I.8)

- la descripción de las características físicas de los personajes implica generalmente una valoración de tipo ético, ya que los ca- 
racteres moralmente aceptables suelen poseer también belleza física:

\begin{abstract}
"And indeed, if Mrs. Tow-wouse had given no Utterance to the Sweetness of her Temper, Nature had taken such Pains in her Countenance, that Hogarth himself never gave more Expression to a Picture" (J.A., 1.14)

"but to comprehend her entirely, conceive Youth, Health, Bloom, Beauty, Neatness, and Innocence in her Bridal-Bed; conceive all these in their utmost Perfection, and you may place the charming Fanny's Picture before your eyes" (J.A., IV.16)

"Mademoiselle de la Rappinière reçut la compagnie avec force compliments, comme elle était la femme du monde qui se plaisait le plus à en faire. Elle n'était pas laide, quoique si maigre et si sèche qu'elle n'avait jamais mouché de chandelle avec les doigts que le feu n'y prit; jen pourrais dire cent choses rares, que je laisse de peur d'être trop long" (R.C., I.4)

"Le Destin et L'Étoile, Léandre et Angélique, deux couples de beaux et parfaits amants..." (R.C., II.17)
\end{abstract}

El uso de generalizaciones de tipo filosófico o moral o de dichos y hechos universalmente aceptados, que sirven de comentario al desarrollo de la acción, es un nuevo signo de la continua presencia del narrador en el relato. Este tipo de intromisiones relacionan los hechos o los personajes de la historia con la vida real, los extraen de su universo de ficción, por lo que puede apreciarse un cierto afán de realismo en semejantes comentarios.

El objeto inmediato de estas generalizaciones es diverso:

- las más abundantes son aquellas que Chatman denomina "filosóficas" y que son la expresión de opiniones comúnmente aceptadas y, por tanto, supuestamente compartidas por el lector:

"As Wit is generally observed to love to reside in empty Pockets..." (J.A., I.12)

"But Fortune, which seldom doth good or ill, or makes Men happy or miserable by halves..." (J.A., IV.15)

"...tant il est vrai qu'il n'y a rien de certain en ce monde" (R.C., I.4)

"L'amour, qui fait tout entreprendre aux jeunes et tout oublier aux vieux..." (R.C., I.19) 
- estas generalizaciones versan a veces sobre el comportamiento humano:

"It a trite but true Observation, that Examples work more forcibly on the Mind than Precepts" (J.A., I.1) "... as the Passion generally called Love, exercises most of the Talents of the Female or fair World..." (J.A., IV.7) “...parce que l'homme le plus sage n'est pas quelquefois maitre de sa langue..." (R.C., I.16)

"Mais comme les femmes se prennent souvent par où elles devraient moins se laisser prendre..." (R.C., II.6)

- ciertos dichos populares constituyen en otros casos la base de estos comentarios:

"Those who have read any Romance or Poetry antient or modern, must have been informed, that Love hath Wings" (J.A., I.11)

“...for Hunger is better than a French Cook" (J.A., III.8)

“...quand le diable, qui ne dort jamais..." (R.C., I.2)

"La Rappinière lui fit cent questions sur la comédie et, de fil en aiguille..." (R.C., I.5)

El narrador de Joseph Andrews o Le Roman Comique no sólo interpreta los hechos de la historia, enjuicia el comportamiento de los personajes o rompe la barrera ficción/realidad con sus habituales comentarios, sino que, además, en un intento supremo de aproximación al lector, parece querer compartir con él su experiencia de relator; así va aparentemente desvelando el entramado básico del arte narrativo, sus propias opiniones sobre teoría literaria, en un juego que no debe engañar al receptor del mensaje ya que la intención última del narrador no es aclaratoria, sino irónica. Su habitual intrusismo le permite organizar el relato a su placer, jugar con el tiempo y el espacio, omitir detalles o extenderse deliberadamente en comentarios ajenos al relato.

He aquí los tipos de comentario referentes al discurso utilizados con más frecuencia:

- justificación o referencia a la omisión de ciertos detalles de la historia: 
"A Dispute arose on this Occasion concerning Evidence, not very necessary to be related here" (J.A., I.15)

"After much tossing and turning in Bed, and many Soliloquies, which if we had no better Matter for our Reader, we would give him..." (J.A., 1.7$)$

"Je ne dirai point si les comédiens plurent autant aux dames du Mans que les comédiennes avaient fait aux hommes; quand j'en saurais quelque chose, je n'en dirais rien; mais, parce que l'homme le plus sage n'est pas quelquefois maitre de sa langue, je finirai le présent chapitre, pour m'ôter tout sujet de tentation" $(R . C$., I.16)

"Quoiqu'un fidèle et exact historien soit obligé à particulariser les accidents importants de son histoire et les lieux où ils se sont passés, je ne vous dirai pas fort juste en quel endroit de notre hémisphère était la maisonnette où Ragotin mena ses confrères futurs..." $(R . C$., Il.16)

- el narrador asegura desconocer ciertos detalles de la historia, lo que refleja en unos casos un aparente afán de verosimilitud -al relatar únicamente lo que afirma conocer- y en otros una evidente intención irónica:

"Indeed, 1 have often been assured by both, that they spent these Hours in a most delightful Conversation: but as I never could prevail on either to relate it, so I cannot communicate it to the Reader" (J.A., II.15)

"This was all of Mr. Joseph Andrews's Speech which 1 could get him to recollect, which I have delivered as near as possible in his own Words, with a very small Embellishment" (J.A., III.6)

"Destin coucha seul en une petite chambre, La Caverne avec la fille de chambre, dans un cabinet et La Rancune avec le valet, je ne sais où" (R.C., 1.4)

"Ils se dirent encore cent belles choses, que je ne vous dirai point, parce que je ne les sais pas et que je n'ai garde de vous en composer d'autre, de peur de faire tort a dom Carlos et à la dame inconnue" (R.C., 1.9)

- el narrador suele advertir al lector de los cambios de escena:

"We shall therefore return to the Lady Booby, and give our Reader some Account of her Behaviour" (J.A., 1.6)

"... where we will leave her and Joseph together for some time, to entertain the Reader with other Matters" (J.A., I.13) 
"Mais il n'y a que trop longtemps que je vous ennuie de la débauche de Ragotin; retournons à l'hôtellerie des comédiens" $(R . C ., 1.11)$

"Nous laisserons courir avec son butin cet homme ... et ne quitterons point Ragotin qui ne veille pas et qui a grand besoin d'être réveillé" (R.C., II.16)

- a menudo se anticipan los acontecimientos en un afán de mantener vivo el interés del lector:

"The Gentleman sat down and Adams by him, and then the latter began, as in the following Chapter, a Discourse which we have placed by itself, as it is not only the most curious in this, but perhaps in any other Book" (J.A., II.7)

"Then Joseph made a Speech on Charity, which the Reader, if he is so disposed, may see in the next Chapter; for we scorn to betray him into any such Reading, without first giving him Warning" (J.A., III.5)

"Il y eut encore dans la même hôtellerie d'autres personnes atteintes du même mal, aussi dangereusement pour le moins que ceux dont je viens de vous révéler le secret; mais nous vous les feron connaitre en temps et lieu" (R.C., I.19)

“...ils étaient dans un gran chemin aisé à suivre et qui les conduisait en un village où nous les allons faire arriver dans le suivant chapitre" (R.C., II.12)

- en otras ocasiones se hace referencia a hechos ya conocidos por el lector:

“...the Reader hath already seen her tender Advances had not met with the Encouragement she might have reasonably expected" (J.A., I.9) "for you may remember, Reader, that the Ravisher had tore her Handkerchief from Fanny's Neck..." (J.A., IV.7)

"Il vous souviendra, s'il vous plaît, que dans le précédent chapitre, l'un de ceux qui avaient enlevé le curé de Domfront avait quitté ses compagnons..." (R.C., I.15)

"Cet homme sauvage fit de grands efforts pour ôter à Ragotin les bottes neuves que... La Rancune s'était appropriées par la supposition des siennes, de la manière que je vous l'ai conté en quelque endroit de cette véritable histoire" $(R . C$., II.16) 
Si la mayor parte de los comentarios relativos al discurso llevan implícita una indudable carga irónica, la máxima expresión de esta ironía y del poder omnímodo del narrador la constituyen las interrupciones a que se ve sometido el relato en ambas novelas; no se trata ya de la inclusión de breves comentarios alusivos a los acontecimientos o a la actitud o carácter de los personajes, sino de la exposición de opiniones o teorías personales, supuestamente relacionadas con algún punto de la historia, pero totalmente gratuitas. Con el evidente propósito de demostrar una vez más su preponderancia, el narrador deja en suspenso el relato durante un lapso de tiempo más o menos largo para ofrecer después una justificación de carácter irónico al lector.

Joseph Andrews ofrece varios ejemplos de este tipo de digresiones; así, por ejemplo, inmediatamente después del rapto de Fanny a manos de los secuaces del "squire" (III.10), se permite dedicar dos capítulos a sendos diálogos de carácter teórico, manteniendo en vilo la curiosidad del lector, a quien convierte además en objeto de su ironía al afirmar:

\begin{abstract}
"Neither the facetious Dialogue which pass'd between the Poet and Player, nor the grave and truly solemn Discourse of Mr. Adams, will, we conceive, make the Reader sufficient Amends for the Anxiety which he must have felt on the account of poor Fanny, whom we left in so deplorable a Condition" (III.12).
\end{abstract}

Es también su inigualable sentido de la ironía lo que impulsa en otro momento al narrador a interrumpir el relato e incluir su grandilocuente repulsa de la vanidad "for no other Purpose than to lengthen a short Chapter" (I.15)

Más expresiva aún de la inefable autoridad del narrador de Le Roman Comique es la cómica interrupción que tiene lugar al final del capítulo 19 cuando Ragotin, en una escena de claras reminiscencias quijotescas, queda literalmente colgado del pomo de la silla de su caballo en una postura realmente ridícula; he aquí el comentario cómicoirónico con que finaliza el capítulo:

“...le malheureux se trouva le pommeau entre les fesses, où nous le laisserons comme sur un pivot pour nous reposer en peu; car, sur mon honneur, cette description má plus coûté que tout le reste du livre et encore n'en suis-je pas trop bien satisfait" (I.19). 
Al comienzo del capítulo siguiente el narrador insiste en la cómica imagen ofrecida por Ragotin para reanudar más tarde el relato tras este preámbulo irónico:

"Je ne crois pas que défunt Phaéton, de malheureuse mémoire, ait été plus empêché après les quatre cheveux fougueux de son pére que le fut alors notre petit avocat sur un cheval doux comme un âne; et, s'il ne lui en coûta pas la vie comme à ce fameux téméraire, il s'en faut prendre à la fortune sur les caprices de laquelle j'aurais un beau champ pour m'étendre si je n'étais obligé en conscience de le tirer vitement du péril où il se trouve" (I.20).

Los títulos de gran parte de los capítulos de ambas novelas constituyen un ejemplo adicional del carácter del narrador; dichos títulos no se limitan a informar del contenido del capítulo, sino que incluyen frecuentemente aclaraciones dirigidas al lector o interpretaciones personales del propio narrador. En Joseph Andrews son muy frecuentes los comentarios irónicos incluidos en estos encabezamientos:

"The Death of Sir Thomas Booby, with the affectionate and mournful Behaviour of his Widow, and the great Purity of Joseph Andrews" (J.A., I.5)

"In which, after some very fine Writing, the History goes on, and relates the Interview between the Lady and Joseph; where the latter hath set an Example, which we despair of seeing followed by his Sex, in this vicious Age" (J.A., I.7)

"Containing as surprizing and bloody Adventures as can be found in this, or perhaps any other authentic History" (J.A., III.9)

"Dans lequel on verra plusieurs chosses nécessaires à savoir pour l'intelligence du présent livre" (R.C., I.8)

"Des moins divertissants du présent volume" (R.C., II.11).

La presencia del narrador no sólo se pone de manifiesto a través de los continuos comentarios de uno u otro tipo repartidos a lo largo del relato; el control ejercido sobre la obra, y en definitiva sobre el lector, es de tal grado que el narrador no muestra reparo alguno en presentarse ante él como el auténtico emisor de muchos de esos comentarios y en aparecer, sin ningún recato, representado por un pronombre en primera persona. A lo largo de este análisis se han ofrecido 
varios ejemplos de este recurso, ya que cualquiera de los tipos de comentario citados puede servir de vehículo para estas intromisiones personales.

El narrador de estas dos novelas es, pues, un típico ejemplo del "narrador dramatizado" -según la terminología de Booth ${ }^{5}$ - que juega un papel de protagonista dentro del relato, desplazando a los mismos personajes. Su figura, aunque quizá poco ortodoxa desde el punto de vista de la crítica actual, ejerce una indudable fascinación en el lector moderno, que no percibe en la misma medida que el contemporáneo de Fielding o Scarron la carga moralizante que invade en muchos momentos sus intervenciones, especialmente en el caso de Joseph Andrews. Esa evidente manipulación del lector de que habla Iser ${ }^{6}$ con referencia a las novelas de Fielding ha perdido eficacia con el paso del tiempo, aunque sin duda se mantienen los presupuestos morales básicos sobre los que estos autores apoyan su afán didáctico -menos marcado aunque no inexistente en Le Roman Comique.

El lector actual percibe, desde luego, esa omnipresencia del narrador, pero no se siente objeto de la misma; este narrador todopoderoso se ha convertido más bien en un ingenioso amigo, cuyas opiniones, siempre inteligentes, son generalmente compartidas con una sonrisa. El lector contemporáneo tampoco se siente objeto de la ironía del narrador; más bien disfruta de ella desde su superior postura de cómplice. En cualquier caso, es innegable que el paso del tiempo no ha invalidado esta figura ni el propósito con el que fue creada, estimular la participación del lector en la obra literaria.

Booth, op.cit., pp. 201 y sig.

6 W. Iser, The Act of Reading, London, Routledge \& Kegan Paul, 1978, pp.152-153. 